\title{
Inhibition of IRS-1 Alters Retinal Circadian Clock
}

\author{
Maaz Arif ${ }^{1}$, Deepa Mathew ${ }^{2}$, Ashay Bhatwadekar ${ }^{2}$ \\ ${ }^{1}$ Indiana University School of Medicine, ${ }^{2}$ Indiana University School of Medicine, \\ Department of Ophthalmology
}

\section{Background and Hypothesis:}

Life on Earth has adapted to a 24-hour cycle of light and darkness. Circadian physiology coordinates temporal metabolism, hormone cycling, and sleep using clock genes. It is documented that dysregulation of clock gene expression is a key factor in the pathogenesis of diabetic retinopathy. Our goal was to explore the relationship between clock genes and the retina in diabetic milieu, and we hypothesized that disrupting downstream insulin signaling in a manner similar to diabetes in the retina would also affect the circadian clock.

\section{Experimental Design or Project Methods:}

In this mouse study, we used a Per2::Luc fusion protein to perform real-time bioluminescent recording of circadian rhythms. We used SecinH3 to inhibit Insulin Receptor Signaling (IRS-1). In isolated retinas, we modulated IRS-1 to mimic the diabetic condition of impaired insulin signaling; this allowed us to directly quantify circadian rhythms in the retina.

\section{Results:}

Our results show that IRS-1 inhibition by SecinH3 altered the gene expression of Per2, a clock regulatory gene, over the controls. There was an increase in the period and an apparent phase shift in the presence of 100uM SecinH3.

\section{Conclusion and Potential Impact:}

Our findings can help us understand the role of insulin signaling on circadian rhythms of the retina and provide another temporal dimension to view diabetic retinopathy disease progression. Ultimately, further studies and a closer understanding of the roles of molecular clocks and insulin signaling may help to develop novel therapeutics for treating some of the harmful effects of diabetes. 